\title{
Protected Turning Movements of Noncooperative Automated Vehicles: Geometrics, Trajectories, and Saturation Flow
}

\author{
Xiaobo Liu $\left(\mathbb{D},{ }^{1}\right.$ Lijuan Lai, ${ }^{1}$ You Kong, ${ }^{1}$ and Scott Le Vine $\mathbb{D}^{1,2,3}$ \\ ${ }^{1}$ School of Transportation and Logistics, Southwest Jiaotong University, Chengdu, China \\ ${ }^{2}$ Department of Geography, State University of New York (SUNY) at New Paltz, New Paltz, NY, USA \\ ${ }^{3}$ Department of Civil and Environmental Engineering, Imperial College London, South Kensington SW7 2AZ, UK
}

Correspondence should be addressed to Scott Le Vine; levines@newpaltz.edu

Received 26 May 2017; Accepted 15 November 2017; Published 10 January 2018

Academic Editor: Emanuele Crisostomi

Copyright (C) 2018 Xiaobo Liu et al. This is an open access article distributed under the Creative Commons Attribution License, which permits unrestricted use, distribution, and reproduction in any medium, provided the original work is properly cited.

\begin{abstract}
This study is the first to quantify throughput (saturation flow) of noncooperative automated vehicles when performing turning maneuvers, which are critical bottlenecks in arterial road networks. We first develop a constrained optimization problem based on AVs' kinematic behavior during a protected signal phase which considers both ABS-enabled and wheels-locked braking, as well as avoiding encroaching into oncoming traffic or past the edge-of-receiving-lane. We analyze noncooperative ("defensive") behavior, in keeping with the Assured Clear Distance Ahead legal standard to which human drivers are held and AVs will likely also be for the foreseeable future. We demonstrate that, under plausible behavioral parameters, AVs appear likely to have positive impacts on throughput of turning traffic streams at intersections, in the range of $+0.2 \%$ (under the most conservative circumstances) to $+43 \%$ for a typical turning maneuver. We demonstrate that the primary mechanism of impact of turning radius is its effect on speed, which is likely to be constrained by passenger comfort. We show heterogeneous per-lane throughput in the case of "double turn lanes." Finally, we demonstrate limited sensitivity to crash-risk criterion, with a $4 \%$ difference arising from a change from 1 in 10,000 to 1 in 100,000,000. The paper concludes with a brief discussion of policy implications and future research needs.
\end{abstract}

\section{Introduction}

Within the functional lifetime of much of today's road infrastructure, vehicle automation is expected to impact a range of important aspects of the transportation system, including traffic operations [1-3], safety [4], public transportation networks [5], design of arterial streets, limitedaccess facilities, parking facilities [6-8], activities performed while traveling [9], and possibly the dominance of the private automobile ownership business model $[10,11]$.

This study fits within the wider body of literature that addresses the issue of how automated vehicles (AVs) will impact traffic flow. This is a broad research question, with far-reaching consequences for future investment in the road network. This study's specific objective is to quantify the impact of AVs on the traffic flow properties of protected turning movements at signalized intersections (i.e., left- or right-turning vehicles performing turning maneuvers during a protected signal phase). We formulate a constrained optimization model of defensive driving car-following behavior during a turning maneuver that takes into account AVs' sensing/processing/actuating capabilities and then employ this model to estimate capacity (vehicles per hour per lane). The intent is to provide both a methodology and numerical results suitable for application in transportation planning models when attempting to forecast the impact of AVs on traffic flow and related outcomes (e.g., emissions). This research is timely, as the state of the art in planning studies attempting to forecast AVs impacts (cf. [12-14]) is to arbitrarily increase roadway network capacity; Guerra [15] reports that "nearly all" transportation plans in the USA do not consider AVs, which he demonstrates (on the basis of practitioner interviews) to be attributable to "uncertainties about the technology." Planners, network managers, and policymakers therefore urgently require estimates of capacity impacts based on fundamental kinematic analysis rather than guesswork. 
Vehicle automation is often analyzed alongside vehicleto-X (V2X) connectivity; the focus of this research is on noncooperative automated vehicles in which the only source of information that an individual automated vehicle uses to make safety-critical driving decisions is line-of-sight information from its own vehicle-borne sensors. While large gains in throughput can in principle be delivered through V2X connectivity, there are major barriers to delivering these benefits and there is evidence of the automotive sector preparing for an extended period of noncooperative driving (see extended discussion in Section 3.1).

Turning maneuvers account for a relatively small proportion of the time that vehicles are in motion on an arterial road network; however, they act as important bottlenecks on network-wide capacity for several distinct reasons [1618]. A first mechanism is that a vehicle approaching a turning maneuver generally must slow as it approaches the location of the maneuver, thereby increasing the duration of the time-slot that it consumes a given physical space and hence reducing capacity. A second mechanism is that, at a typical four-way intersection, left-turning vehicles compete for signal time with through traffic and right-turning vehicles traveling in the opposing direction, in addition to traffic on the cross street. Finally, both left-turning and right-turning vehicles at arterial road-network intersections conflict with pedestrians, introducing a third mechanism that constrains capacity.

This study quantifies saturation flow rates for AVs performing turning maneuvers and is subject to a set of limitations. First, our focus is on protected turning movements; we leave the issue of "permitted" turning movements (i.e., movements that involve accepting a gap in a conflicting traffic stream) as a matter for future research, along with issues of sight line limitations and interactions between AVs and pedestrians (cf. [3]). Second, by focusing on saturation flow (i.e., maximum sustained flow), we explicitly do not address the decreased-flow regimes (termed "start-up" and "clearance" in [19]) that occur at the beginning and end, respectively, of a signal phase when vehicles are not proceeding at a steady-state rate. Third, we focus on defensive driving (i.e., noncooperative) behavior, as described in Section 3; this is the standard to which human drivers are held. More aggressive driving strategies such as short-headway platooning of connected AVs would tend to increase capacity; however, this behavior would be fundamentally inconsistent with the longstanding responsibility of the operator of each vehicle to not strike (i.e., "rear-end") the vehicle ahead of it.

The remainder of this paper is organized as follows: Section 2 presents further background relevant to this study. Section 3 introduces the data and methods employed in the analyses. Section 4 presents and discusses the empirical calculations of saturation flow rates, and Section 5 concludes the paper.

\section{Background}

Given the major policy and operational issues surrounding the issue of road-network capacity, a wide and growing body of literature has attempted to quantify the potential capacity impacts of automated vehicles. There is much interest in, for instance, whether AVs may enable increased capacity within the existing footprint of the road network, which would have the potential to reduce congestion and increase journey-time reliability without additional land consumption. A fundamental limitation of the existing literature is that, due to AVs being in their embryonic phase, the general approach to estimating capacity impacts is to employ simulation techniques rather than to perform empirical field testing on closed courses or collect observations from realworld network operations.

The body of literature on AVs' impacts on roadway network capacity (cf. [20] for a review of literature published up to year 2013) can be organized on the basis of whether the context is a freeway environment [21-25] or an arterial environment [26-30], or both [31]. The gap in the literature that this study addresses is that previous studies of AVs capacity impacts have not specified driving behavior during turning maneuvers on the basis of turning vehicles' unique kinematics.

A related body of literature that has taken shape in recent years addresses the AV motion-planning problem (i.e., programming an AV's trajectory as it performs driving tasks); compare [32] for a detailed review of this literature, with Alhajyaseen et al. [33], Gu et al. [18], and Wolfermann et al. [34] having studied the trajectories of turning vehicles at intersections. Studies in this category have focused on the task of controlling the trajectory of a single AV and do not consider the capacity impacts arising from the interactions among multiple AVs' driving behaviors (e.g., consequences on saturation flow rates).

Therefore, to the best of the authors' knowledge this study represents the first in-depth evaluation of the capacity of turning maneuvers under automated driving. Indeed, Hoogendoorn et al. [20], providing a wide-ranging year-2014 review of the literature on AVs and traffic efficiency, reported that "no studies were found on the influence of automation of the lateral control task on traffic flow efficiency" (p.114).

Further, in contrast to earlier studies of AVs kinematics which have specified deterministic braking system performance, we operationalize the "defensive driving" requirement with an analysis that explicitly accounts for the stochastic nature of braking system performance (i.e., achieved deceleration rates that cannot be known a priori with certainty) and which therefore allows us to perform sensitivity tests with a range of plausible crash-risk criteria.

For human drivers, the Highway Capacity Manual [19] is the standard traffic flow model of turning vehicles employed in the USA. Both right- and left-turning vehicles are specified to have lower saturation flow rates than vehicles traveling straight through an intersection, by $5 \%$ and $18 \%$, respectively (see Equations 19-13 and 19-14 of [19]). In this model of intersection operations, turning radius is not explicitly taken into account in calculations of saturation flow rate. However, the lower flow rate of right-turning vehicles is an implicit recognition of the influence of turning radius, given that ceteris paribus a right-turning movement at a 90degree intersection has a smaller radius than a left-turning 
movement (in a right-hand-drive road network). The work of [19] also contains explicit accommodation of the influence of turning radius on throughput in the special case of interchange-ramp-terminal intersections, with smaller turn radii associated with lower flow rates (see Exhibit 23-27). For instance, turning movements at ramp terminals with radii of 25,50 , and 75 feet are defined to have $18 \%, 10 \%$, and $7 \%$ less capacity than through movements, ceteris paribus. A further implicit recognition in [19] of the effect of turn radii is the specification that when multiple turn lanes are present capacity per lane is reduced relative to when a single turn lane exists (by $3 \%$ and $12 \%$ for double-left-turn lanes and doubleright turn lanes, resp.; cf. Exhibit 31-39). Empirically, Cooner et al. [35] studied the operations of triple-left-turn lanes; the results with respect to turn radii were inconclusive, with some sites showing higher capacities for larger-radius turn lanes and some for the smaller-radius lanes. Earlier studies by Sando and Mussa [36], Courage et al. [37], and Leonard [38] reported similarly inconclusive findings regarding the effects of turn radius on capacity of triple-left-turn lanes; however, Ackeret [39] reported higher saturation flow rates for largerradius turn lanes.

\section{Methods}

The analysis strategy for this study incorporated a microsimulation model of the operation of two vehicles (one leading $\left[\mathrm{veh}_{l}\right]$ and one following $\left[\mathrm{veh}_{f}\right]$ ) negotiating a 90-degree turning maneuver. The analysis is discrete in time (0.01second time steps) and continuous in space. Notations are summarized in the Summary of Notations.

3.1. Calculation of Minimum Safe following Headway. Calculating minimum safe following headway for turning vehicles is a more complex problem than the same calculation for vehicles traveling only longitudinally. In the latter case, (1) can be used to determine the minimum safe following distance $x_{\min }$ that allows a following vehicle $\left(\mathrm{veh}_{f}\right)$ to avoid striking the "leading" vehicle $\left(\mathrm{veh}_{l}\right)$ that it is following in the event of $\mathrm{veh}_{l}$ unexpectedly initiating emergency braking:

$$
\begin{aligned}
x_{\min } & =v * t_{\mathrm{lag}, f}+\frac{v^{2}}{2 * a_{f}}-\frac{v^{2}}{2 * a_{l}}+x_{\mathrm{veh}}, \\
H_{\min } & =t_{\mathrm{lag}, f}+\frac{v}{2 * a_{f}}+\frac{x_{\mathrm{veh}}-v^{2} /\left(2 * a_{l}\right)}{v}, \\
C_{v} & =\frac{1}{H_{\min }} .
\end{aligned}
$$

Equations (1) and (2) arise from the equations governing translational motion; the derivation can be found in the Appendix of [30]. Equation (3) expresses capacity $\left(C_{v}\right)$ as the reciprocal of the minimum headway value. In (1), the first term on the right-hand side (incorporating velocity $v$ and latency time $t_{\mathrm{lag}, f}$ ) represents the distance that $\mathrm{veh}_{f}$ travels after veh ${ }_{l}$ begins braking and before $\mathrm{veh}_{f}$ does so. The second term accounts for the distance that $v^{2} h_{f}$ will travel while it brakes. The third term accounts for the distance that $\mathrm{veh}_{l}$ will travel during its braking maneuver and is relevant only for the "weak" ACDA interpretation. This is because under the "strong" interpretation of ACDA it is assumed that $\mathrm{veh}_{l}$ will decelerate at a rate $\left(a_{l}^{-}\right)$that is arbitrarily large, thereby effectively removing this term from the analysis. Finally, the fourth term $\left(x_{\text {veh }}\right)$ represents vehicle length. (Note. Equations (1) and (2) apply only under the conservative assumption that the rate of deceleration of the leading vehicle $\left(a_{l}\right)$ is greater than or equal to the rate of deceleration of the following vehicle $\left(a_{f}\right)$; that is, $a_{l} \geq a_{f}$.)

Equations (1)-(3) formalize the Assured Clear Distance Ahead (ACDA) legal standard, which codifies the driving behavior colloquially termed Defensive Driving. ACDA requires that a car's operator leave sufficient spacing behind the vehicle or object ahead of it, such that it can avoid striking the vehicle/object. In formally defining ACDA, Buchwalter et al. ([40], Automobiles and Highway Traffic, Sect. 1115) report that "most jurisdictions follow the rule that a collision between a preceding and a following motorist gives rise to a presumption of negligence on the part of the following motorist, whether the preceding vehicle is moving, stopped, or stopping." Though ACDA is generally applicable as a criterion, it has not been universally applied to establish negligence in a crash. A vehicle operator that strikes an object that has suddenly "darted out" (i.e., moved laterally) into their trajectory of travel is, for instance, generally not deemed to be negligent ([40], Automobiles and Highway Traffic, Sect. 498). Likewise, ACDA has not been applied uniformly by the judiciary; courts have variously interpreted it "weakly" (cf. Naffky [41], Sect.664) or "strongly," with the distinction that under the "weak" interpretation a driver is held responsible only to avoid striking the vehicle in front of itself that is traveling in the same direction, and under the "strong" interpretation a driver is responsible to avoid striking a stationary object (cf. [42]). The "strong" interpretation of ACDA results in larger following distances and hence larger headways between vehicles, to account for the fact that a vehicle's forward sight line is blocked by the vehicle in front of it. Though there is a wide body of literature that has considered cooperative automated driving (cf. $[2,25,43]$ ), there is also evidence of automotive manufacturers preparing for an extended period of noncooperative automated driving. In a patent application disclosed by Ford Global Technologies (a subsidiary of Ford Motor Company) in 2016, for instance, Stanek and Lockwood [44] envision AVs engaging in ACDA-compliant (noncooperative) driving behavior: "Each of [an AV's] onboard sensors has a limitation in the range (i.e. distance) at which objects and/or conditions can be detected... the top speed at which the vehicle is controlled to travel under autonomous driving mode may be calculated as a factor of how far ahead the sensors can detect objects" (p.3).

We now introduce the additional dimension of lateral (turning) motion, which is not considered in (1)-(2). Figure 1 schematically depicts the position of two vehicles engaged in left-turn maneuvers of fixed-radius $r$ (measured from center-of-circle to vehicle center-of-gravity, consistent with AASHTO 2011 [[45]; cf. Equation (3)-(6)] in sequence ( eh $_{l}$ first, followed by $v e h_{f}$ ). When traveling along a trajectory 


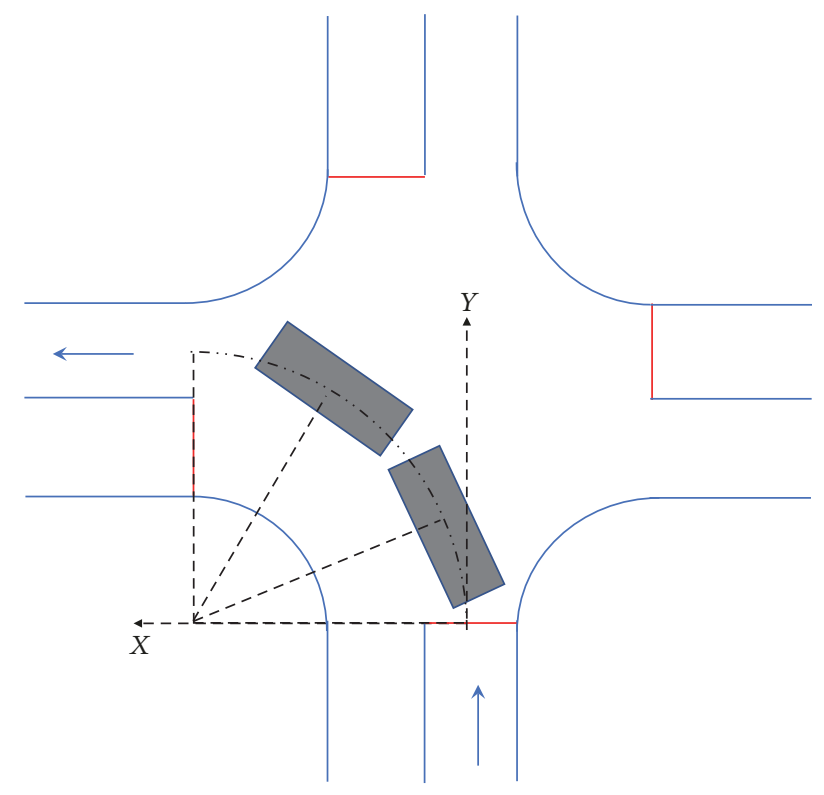

FIGURE 1: Schematic diagram of leading $\left(\mathrm{veh}_{l}\right)$ and following $\left(\mathrm{veh}_{f}\right)$ left-turning vehicles.

which is an arc of a circle, each vehicle's velocity has two components: translational and rotational.

The translational component is similar in some respects to longitudinal-only deceleration when two vehicles following one another both brake in a straight line. However, the difference in the case of turning motion is that the two vehicles are not traveling in sequence in a straight line but rather are at different points along the arc of a circle. The trajectory that the vehicles will follow after initiating emergency braking will depend on whether the braking is "wheel-locked" or is "ABS-engaged" (Automated Braking System), as is the case with new automobiles sold today in the USA (we assume that deceleration occurs at a constant linear rate, consistent with earlier models of emergencybraking maneuvers (cf. $[45,46]$ ) and field testing results of autonomous emergency-braking systems [47]). Steering control is lost for the duration of wheel-locked braking; ABS is designed to preserve steering control (whether braking is initiated while traveling in a straight trajectory or during a turning maneuver), which is achieved by pulsing the brakes more quickly than humans' physical capabilities permit. However, ABS does not in general result in a reduction in achieved braking distance (cf. $[46,48]$ ).

In an ABS-equipped human-driven vehicle, $A B S$ engages automatically during emergency braking. In an AV, however, the vehicle's controller would have much better situational awareness than today's ABS systems and would therefore in principle be able to select which of "wheels-locked" or "ABS-engaged" braking is optimal in any given emergency maneuver (rather than assuming, as ABS systems do with human drivers, that engaging $\mathrm{ABS}$ is always optimal). In the case of two AVs ( $\operatorname{veh}_{l}$ and $\mathrm{veh}_{f}$ ) performing turning maneuvers, there are two separate issues: whether veh would $_{l}$ engage ABS upon initiating emergency braking (which veh ${ }_{f}$ cannot know in advance or control) and whether veh would engage ABS (which veh $\mathrm{v}_{f}$ does control). The consequence of "wheels-locked braking" is that the vehicle's translational motion will continue along the line tangent to the arc of the vehicle's prior trajectory at the point when emergency braking is initiated. By contrast, a vehicle performing ABSengaged braking will continue its translational motion along the curved trajectory until it comes to a rest. In the case of wheels-locked braking, there is an additional constraint that the braking vehicle must not travel into the crosswalk (or past the stopbar if no crosswalk is present) or impact the curb of the exit leg of the intersection during its braking maneuver. As a practical matter, this means that, considering a northbound left-turning vehicle, the vehicle must ensure that if it must initiate emergency braking it will not encroach further north than the northern curbline of the western leg or its eastward extension across the southern boundary between the intersection "box" and the intersection's northern leg.

With regard to rotational motion, we specify constant deceleration, resulting in the rotational orientation at rest $(\alpha)$ of a vehicle during an ABS-enabled braking maneuver calculated via

$$
\alpha=\frac{\omega * t_{\text {braking }}}{2},
$$

where $\omega$ is the initial rotational velocity (expressed in radians or degrees per second) and $t_{\text {braking }}$ is the duration of the braking maneuver, which is equal to the duration required for the translational motion to come to rest.

Figures 2 and 3 show a time-lapse progression of veh $_{l}$

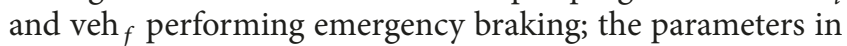
this diagram are radius $r=50^{\prime}, v=20 \mathrm{mph}$, and angle $\beta$ of veh $\mathrm{v}_{l}$ when it initiates emergency braking of 45 degrees. Figures 2 and 3 depict the two vehicles performing ABSengaged and wheels-locked braking maneuvers, respectively. The time-frames shown represent the following:

(i) Top-left: initiation of emergency braking by veh $(t=$ 0)

(ii) Top-right: after a period of time $t_{\text {lag, } f}$ elapses (specified to be 0.4 seconds; see Section 3.2), veh $f$ recognizes that $\mathrm{veh}_{l}$ has initiated emergency braking and also begins emergency braking $(t=0.40$ seconds)

(iii) Bottom-left: $v^{2} h_{l}$ has come to rest ( $t=0.97$ seconds)

(iv) Bottom-right: veh $_{f}$ has also come to rest $(t=1.52$ seconds). Note that in both Figures 2 and 3 veh $_{f}$ has selected its following distance at time $t=0$ to ensure that after the emergency-braking maneuvers it comes to rest at the precise critical location where its front bumper is infinitesimally close to the rear bumper of $\operatorname{veh}_{l}$.

In contrast to the translation component of motion (discussed in the previous three paragraphs), the rotational component describes the type of motion in which a vehicle pivots around a point (in this study, we assume that the rotational motion of each vehicle is centered on the centroid of the vehicle. In practice, the rotational motion of an 


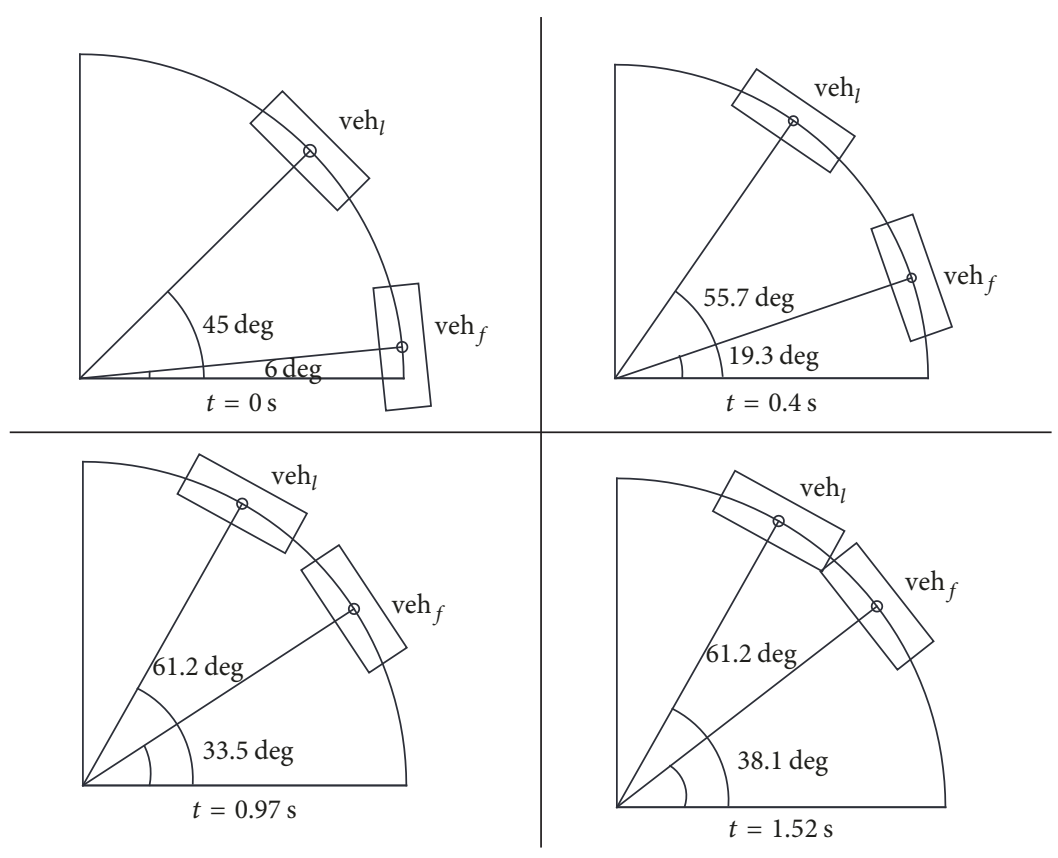

FIGURE 2: Time-lapse sequence of two left-turning vehicles, with $A B S$-engaged braking trajectories. Parameters: $r=50^{\prime}, \beta_{l}=45^{\circ}$, and $v=20 \mathrm{mph}$.

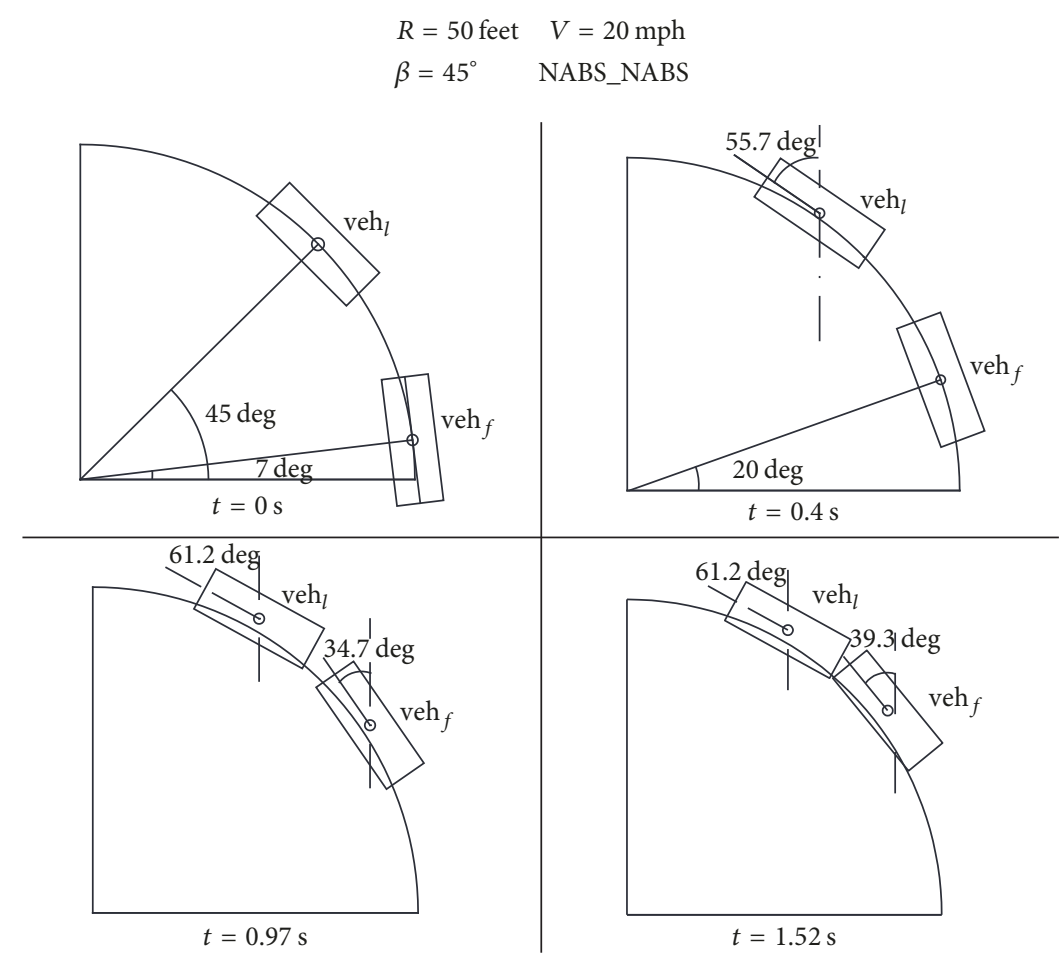

Figure 3: Time-lapse sequence for two left-turning vehicles, with wheels-locked braking trajectories. Parameters: $r=50^{\prime}, \beta_{l}=45^{\circ}$, and $v=20 \mathrm{mph}$.

automobile that is not heavily loaded with passengers or cargo is likely to be located closer to the front of the vehicle, due to the heavy weight of the engine and other vehicular systems located in front of the passenger compartment). When a vehicle is performing a turning maneuver, it changes orientation and therefore experiences rotational or angular velocity (degrees per unit time). Upon initiating emergency braking, this angular velocity will begin to decrease until it reaches zero. As with the translational component of motion, we specify that the rotational component also decelerates at 


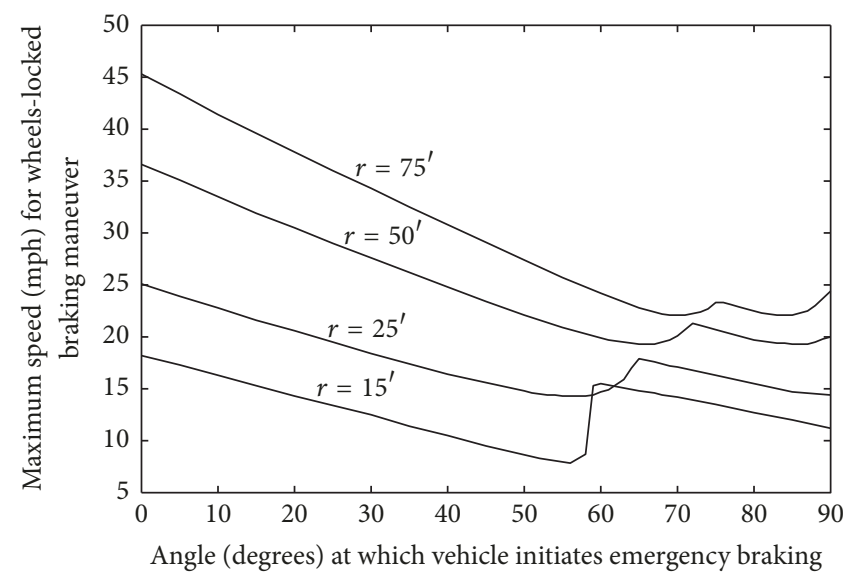

FIGURE 4: Curves depicting maximum speed $\left(v^{\mathrm{WLB}}\right)$ at which a wheels-locked braking maneuver can be performed.

a constant rate, and also that the two components of motion reach zero velocity at the same time (in this study, we assume that the rotational motion of each vehicle is centered on the centroid of the vehicle. In practice, the rotational motion of an automobile that is not heavily loaded with passengers or cargo is likely to be located closer to the front of the vehicle, due to the heavy weight of the engine and other vehicular systems located in front of the passenger compartment). The

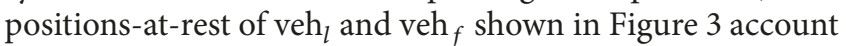
for both components of motion. In general, for left-turning vehicles the wheels-locked-braking position-at-rest will be located above and to the right of the position-at-rest of ABSengaged-braking; this is because the ABS-engaged trajectory involves the same amount of overall motion, but with the $x / y$-axis components of motion being distributed to a larger extent towards the left (following the arc) and therefore to a smaller extent along the $y$-axis towards the top of the graphic.

veh $_{f}$ is the member of this vehicle-couplet that makes the choice of following distance. veh $f$ also has the power to decide whether it will follow the ABS-engaged or wheelslocked trajectory, but it does not know and cannot control which of these two trajectories veh $\mathrm{v}_{l}$ will follow. Therefore, veh $_{f}$ must assume that $\mathrm{veh}_{l}$ follows the "worst-case" of the two trajectories (i.e., the trajectory that would require that veh $_{f}$ leave the largest spacing ahead of it). Because veh has the power to decide which trajectory it will follow, we specify that it selects the minimum of the two safefollowing-distances associated with the ABS-engaged and wheels-locked trajectories. The exception to this is when multiple turn lanes exist: if the two vehicles are in any turn lane except the lane directly adjacent to through traffic in

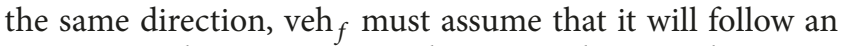
ABS-engaged trajectory in order to avoid encroaching into the adjacent turn lane.
Figure 4 shows the relationship between $\beta$ and the maximum speed $\left(v^{\mathrm{WLB}}\right)$ at which a vehicle can perform a "wheels-locked" braking maneuver without encroaching into oncoming traffic or past the edge-of-lane after exiting the intersection, for selected turning radii $\left(r=15^{\prime}, 25^{\prime}, 50^{\prime}\right.$, and $75^{\prime}$ ). Two general relationships can be seen: $v^{\mathrm{WLB}}$ tends to decrease as $\beta$ increases, and $v^{\mathrm{WLB}}$ also tends to decrease as $r$ decreases. Neither of these relationships is monotonic, however, which is due to the rectangular vehicle shape. Figure 5 demonstrates this phenomenon, by showing the vehicle kinematics that result in the curve for $r=15^{\prime}$ in Figure 4 crossing the curve for $r=25^{\prime}$ in the vicinity of $\beta=60^{\circ}$. At the timeframe ( $t=0.07$ seconds) depicted in both panels of Figure 5, a vehicle traveling initially at $v=$ $15.2 \mathrm{mph}$ would encroach past the edge-of-lane with its frontright corner if $r=25^{\prime}$ (right panel) but would not encroach past the edge-of-lane if $r=15^{\prime}$ (left panel). In the latter case, the rear-right corner of the vehicle would be the first point of the vehicle to encroach past the edge-of-lane if speed were higher.

During a turning maneuver, a vehicle's speed is constrained by two additional factors which do not affect purely longitudinal motion: the amount of friction available to enable the vehicle to successfully navigate the desired curved trajectory, and the comfort of the vehicle's occupant(s) under lateral acceleration, which is proportional to velocity squared (cf. [29]) (for completeness, we note that a third constraint on turning-vehicle speed is the positive relationship between speed and the propensity of the vehicle to "rollover").

We formulate car-following behavior as a constrained optimization problem. Conditional on a set of input parameters $\left(r, v, a_{l}, a_{f}, t_{\mathrm{lag}, f}\right.$, and $\left.x_{\mathrm{veh}}\right)$ that are held constant during a given instance of the optimization process, headway is minimized $\left(H_{\mathrm{min}}\right)$ :

$$
H_{\text {min }}=\min \left\{\max \left\{H_{\min }^{\mathrm{ABS}, \mathrm{ABS}}, H_{\min }^{\mathrm{noABS}, \mathrm{ABS}}\right\}, \max \left\{H_{\min }^{\mathrm{ABS}, \mathrm{noABS}}, H_{\min }^{\mathrm{noABS}, n o A B S}\right\}\right\}
$$



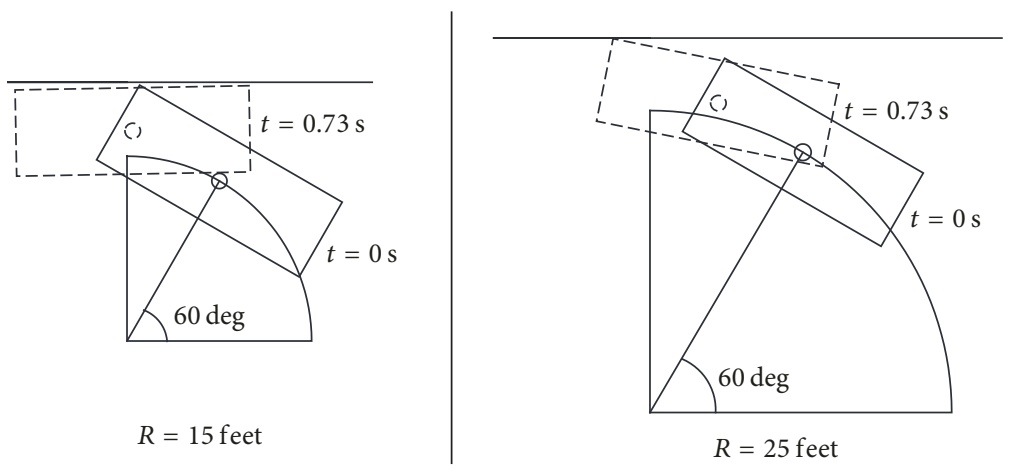

Figure 5: Two time-lapse sequences; each panel depicts a single vehicle's wheels-locked braking maneuver. Parameters: $r=15^{\prime}(\mathrm{L})$ and $r=25^{\prime}(\mathrm{R}), \beta=60^{\circ}$, and $v=15.2 \mathrm{mph}$. Solid-outline vehicle and dashed-outline vehicle depict location at time of $t=0$ and $t=0.73 \mathrm{~seconds}$, respectively.

subject to the following constraint:

$$
\prod_{\beta=0}^{90^{\circ} \infty} \prod_{t=1}^{\infty} I_{\beta t}=1 .
$$

The logic of the minimum and maximum functions in the objective function (see (5)) is explained earlier in this section. In Constraint (6), $I_{\beta t}$ is an indicator function that takes a

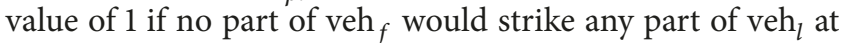

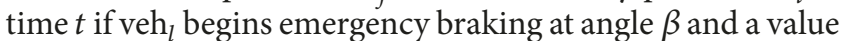
of 0 otherwise. The overall product function (evaluated across all values of $\beta$ and $t$ ) defined by Constraint (6) therefore takes a value of 1 if and only if a candidate headway value is large enough to ensure that veh $\mathrm{h}_{f}$ can be assured (conditional on the input parameters) of not striking $v^{2} h_{l}$ regardless of the possibility of noncooperative driving behavior by $\mathrm{veh}_{l}$ at any point during the turning maneuver. Any candidate headway value that results in Constraint (6) evaluating to 0 is therefore rejected as "following too closely." The constrained optimization problem was solved through simulation due to the objective function not being continuously differentiable.

3.2. Values of Empirical Parameters. Several parameters in (1) require empirical specification in order to calculate the capacity of AVs performing turning maneuvers.

The latency time $\left(t_{\mathrm{lag}, f}\right)$ of AVs' sensing/processing was specified to be 0.4 seconds, as in [30]. We note that assumptions of smaller values of (e.g., 0.2 seconds) would require sustained favorable visibility and weather conditions and $0 \%$ failure rates in sensing/control [4] and that larger values of latency will limit the number of instances that emergency braking is unnecessarily initiated, because a larger number of sensor readings become available.

We specified cars' length and width to be 19 feet and 7 feet, respectively, per the "passenger car" design vehicle in [45].

The deceleration values of the two vehicles ( $a_{l}$ and $a_{f}$ for $\mathrm{veh}_{l}$ and $\mathrm{veh}_{f}$, resp.) are drawn from empirical testing of ABS-enabled vehicles performed by the National Highway Traffic Safety Administration [49]. Empirical results of emergency-braking test runs of a Chevrolet Malibu passenger sedan on four days of dry conditions were employed (cf. [49] Tables E-4, F-4, G-4, and H-4). Of 50 braking maneuvers performed during the testing in [49], we removed 16 due to insufficient pressure applied to the brake pedal. Of the remaining 34 braking maneuvers with acceptable brakingpedal pressure, we first calculated the mean $\left(28.3 \mathrm{ft} / \mathrm{sec}^{2}\right)$ and standard deviation of the rate of deceleration $\left(0.67 \mathrm{ft} / \mathrm{sec}^{2}\right)$. We then tested the distribution for normality using the Shapiro-Wilk test, which supported the null assumption that the data are normally distributed, and subsequently developed estimates of various percentile points of the distribution. We specified $a_{l}$ to be at the 99.9th percentile of the distribution $\left(-30.38 \mathrm{ft} / \mathrm{sec}^{2}\right)$ and the deceleration rate of $a_{f}$ to be at the 0.1 th percentile $\left(-26.21 \mathrm{ft} / \mathrm{sec}^{2}\right)$. The joint probability of these two rates being exceeded (in the case of $a_{l}$ ) or not achieved (in the case of $a_{f}$ ) is $1 / 1,000,000$. In Section 4, we present sensitivity analyses to take into account the possibility that AVs could be programmed to take either larger or smaller risks than this criterion. We note that achievable deceleration rates during emergency braking depend on vehicular technology, which has evolved since the braking tests reported in [49] were performed in the year 2000 and is expected to continue to evolve as increasing levels of automation influence future vehicle design.

In the next section, we present results from a range of combinations of turn radius and travel speed. While alternative specifications are possible (cf. [29] regarding the relationship between lateral acceleration and occupant comfort within $\mathrm{AVs}$ ), for the purposes of direct comparison of AV turning-movement saturation flow to human drivers we specified that AVs select their turning velocity by emulating human drivers' behavior. In the empirical analysis presented in Section 4, we characterized this behavior using the model of the relationship between radius and turning speed proposed by Wolfermann et al. [34]; for the four turning radii studied in Section $4\left(r=15^{\prime}, r=25^{\prime}, r=50^{\prime}\right.$, and $r=75^{\prime}$ ), this turning speed (denoted by $v^{\mathrm{HDM}}$ ) is 12.4, $12.9,14.0$, and $15.2 \mathrm{mph}$, respectively. 


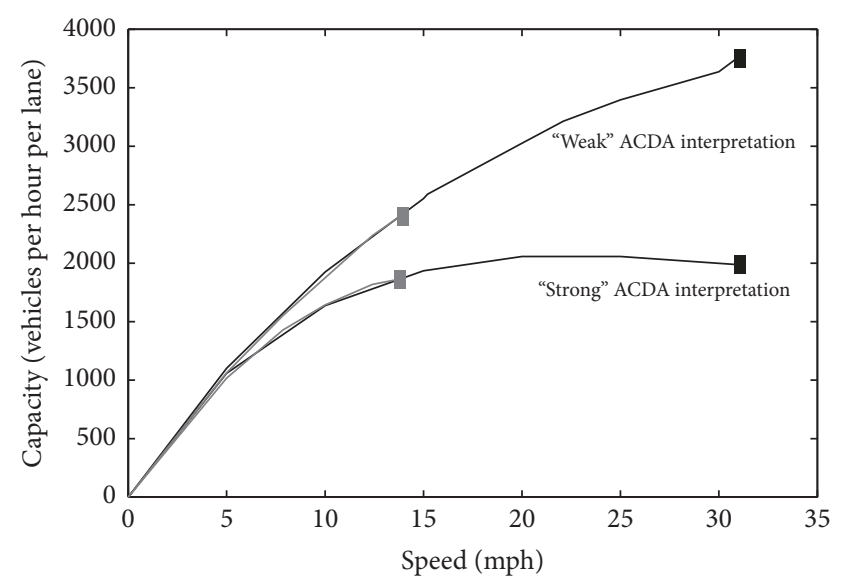

FIGURE 6: Relationship between speed, turning radius, and throughput (vehicles per hour). Gray and black curves represent $r=15^{\prime}$ and $r=75^{\prime}$, respectively; square markers indicate $v^{\mathrm{CTF}}$.

\section{Empirical Calculations of Saturation Flow Rates}

Figure 6 shows the fundamental diagrams of traffic flow (speed plotted versus capacity) of turning vehicles based on the "weak" and "strong" ACDA interpretations (see Section 3.1). Of the four turning radii $\left(15^{\prime}, 25^{\prime}, 50^{\prime}\right.$, and $\left.75^{\prime}\right)$ that we analyzed, only the smallest $\left(15^{\prime}\right)$ and largest $\left(75^{\prime}\right)$ values are shown. Figure 6 shows that the effect of turning radius by itself is small, as the curves for $r=15^{\prime}$ and $r=75^{\prime}$ are nearly indistinguishable. The square markers in Figure 6 depict the maximum turning speed $v^{\text {CTF }}$ for both turning radii that can be performed without skidding off of the turning-arc trajectory, due to the limitation of friction at the tire/roadway-interface (calculated to be a friction factor of 0.85 , using the empirical value of $a=28.3 \mathrm{ft} / \mathrm{sec}^{2}$; cf. Section 3.2). The gray and black square markers in Figure 6 represent the $v^{\mathrm{CTF}}$ values for $r=15^{\prime}\left(v^{\mathrm{CTF}}=13.8 \mathrm{mph}\right)$ and $r=75^{\prime}\left(v^{\mathrm{CTF}}=30.9 \mathrm{mph}\right)$, respectively. Here we see that, under the "weak" ACDA interpretation, the saturation flow rate at $r=75^{\prime}$ (3750 veh/hour/lane) is much larger than the saturation flow rate for $r=15^{\prime}$ (2400 veh/hour/lane), though this difference is much smaller (both absolutely and proportionately) for the "strong" ACDA interpretation (1989 versus $1865 \mathrm{veh} /$ hour/lane). Therefore, it can be concluded that the principal constraint on capacity of AVs performing turning maneuvers at intersections is the programmed turning speed (which is influenced by turning radius), rather than turning radius itself, and that the ultimate constraint on turning speed is therefore likely to be passenger comfort under lateral acceleration.

Table 1 extends from Figure 6, by presenting capacity calculations for both interpretations of ACDA, four turning radii $\left(r=15^{\prime}, 25^{\prime}, 50^{\prime}\right.$, and $\left.75^{\prime}\right)$, three turning speeds $\left(v^{\mathrm{WLB}}, v^{\mathrm{HDM}}\right.$, and $v^{\mathrm{CTF}}$, calculated separately for each turning radius), and two "lane contexts." Lane context refers to whether a single turn lane exists to serve a turning movement or whether multiple turn lanes are present. In the case of multiple turn lanes operating at capacity, vehicles in all turn lanes except the largest-radius turn lane (i.e., the lane directly adjacent to the "through" lanes of the same direction of travel) have the constraint that they cannot perform a "wheels-locked" braking maneuver that would intrude into the adjacent turn lane and therefore must brake along the "ABS-engaged" trajectory. It can be seen that for all combinations of radius and speed the "weak" ACDA interpretation results, as expected, in higher capacity than the "strong" interpretation. Table 1 also shows that, with turning speeds equal to the estimated average speed of human drivers negotiating the same turning maneuver (i.e., $v^{\mathrm{WLB}}$ ), the calculated saturation flow rates consistently represent an increase in capacity relative to human drivers across all four radii. For $r=25^{\prime}$, for instance, this increase varies between a minimum of $+0.2 \%$ (for a left turn, strong ACDA interpretation, and the presence of multiple turn lanes: 1809 versus $1850 \mathrm{veh} /$ hour/lane) to a maximum of $+43 \%$ (for a right turn, weak ACDA interpretation, single turn lane: 2308 versus 1615 veh/hour/lane). The largest calculations of capacity occur at the highest speeds conditional on available friction $\left(v^{\mathrm{CTF}}=\right.$ $30.9 \mathrm{mph}$, for $\left.r=75^{\prime}\right)$; headways less than one second $(0.96$ seconds, equating to saturation flow of $3750 \mathrm{veh} /$ hour/lane) can theoretically be sustained.

The analysis up to this point has specified 1 crash in $1,000,000$ as the criterion for acceptable crash risk if $v h_{l}$ initiates emergency braking. Table 2 shows the results of a sensitivity analysis in which this criterion is varied by two orders of magnitude both larger and smaller, for a moderateradius turning movement ( $r=25$ feet) with a single turn lane and under the weak ACDA interpretation. It can be seen that capacity is relatively insensitive to the choice of safety standard: varying the crash risk by four orders of magnitude (between a 1 in 10,000 risk and a 1 in 100,000,000 risk) leads to only a $4 \%$ variation in turning-movement capacity (2857 versus 2748).

\section{Conclusions}

This study represents the first systematic evaluation of the traffic flow properties of automated vehicles when performing turning maneuvers. The driving behavior model respects the Assured Clear Distance Ahead criterion, which codifies "defensive driving" practice. We evaluate the kinematics of two distinct types of braking maneuvers for collision avoidance and specify that the AV trajectory-planning incorporates for the maneuver that permits the shortest following distance behind the leading vehicle while respecting the ACDA standard.

Our results demonstrate that the impact of AVs on the capacity (vehicles per hour per lane) of turning movements will be constrained primarily by the comfort of vehicle occupants (as represented in this analysis by the typical turning speed of human drivers as a function of turning radius), rather than AVs' performance limitations or the available friction at the tire/roadway-interface. We show that the rectangular shape and typical size of a passenger car lead to complex relationships between turning radius and feasible 


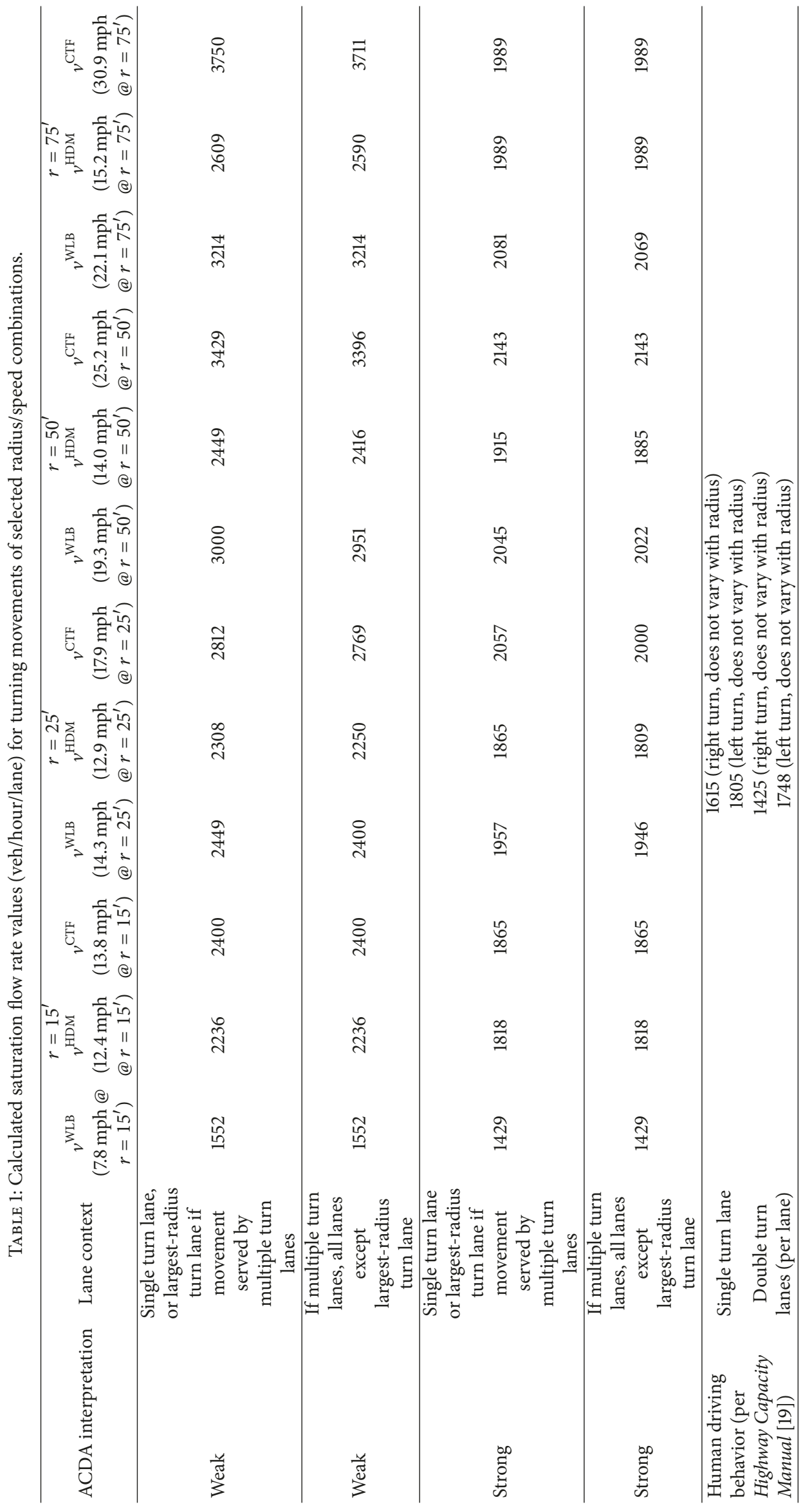


TABLE 2: Sensitivity analysis of rates of deceleration of leading and following vehicle (weak ACDA interpretation).

\begin{tabular}{lccc}
\hline $\begin{array}{l}\text { Crash-risk } \\
\text { criterion }\end{array}$ & $a_{l}\left(\mathrm{ft} / \mathrm{sec}^{2}\right)$ & $a_{f}\left(\mathrm{ft} / \mathrm{sec}^{2}\right)$ & $\begin{array}{c}\text { Capacity } \\
r=25^{\prime}, \\
v^{\mathrm{CTF}}=17.9 \mathrm{mph}\end{array}$ \\
\hline 1 in 10,000 & -29.87 & -26.73 & 2857 \\
1 in 100,000 & -30.14 & -26.46 & 2839 \\
1 in $1,000,000$ & -30.38 & -26.21 & 2812 \\
1 in & -30.60 & -26.00 & 2801 \\
$10,000,000$ & & & \\
1 in & -30.81 & -25.79 & 2748 \\
$100,000,000$ & & & \\
\hline
\end{tabular}

vehicle kinematics. We demonstrate that, as with human drivers, for some combinations of speed and turning radius adding additional turning lanes to serve AVs performing a turning movement would be subject to diminishing marginal returns (i.e., doubling the number of lanes from one to two would lead to less than a doubling of capacity). Finally, we demonstrate that the capacity estimates are relatively robust with regard to selection of a quantitative standard for safety; capacity is shown to vary by $4 \%$ in response to varying the safety standard by four orders of magnitude (i.e., a factor of 10,000 , between a 1 in 10,000 risk and a 1 in 100,000,000 risk).

We now conclude with a brief discussion of research needs for the next phase of the research agenda.

First, we show that achievable flow rates of AVs performing turning maneuvers will have a strong dependence on AVs' turning speed, which implies that establishing guidelines for determining turning speeds may be an important policy consideration. For human drivers, turning speed is a decision made by each individual driver during each specific turning maneuver, subject only to the external constraint of a speed limit. Capacity is maximized by fast turning speeds; however individual AV occupants may desire the low rates of lateral acceleration achieved through slow turning speeds [29]. There may therefore be a role for public policy in specifying the envelope of acceptable turning speeds and rates of lateral acceleration/deceleration in a range of different circumstances. For instance, Urmson et al. [50] proposed an AV-control paradigm through which "an autonomous vehicle may be configured with some general safe envelope driving patterns... adaptive autonomous driving [then] allows the vehicle to perform autonomous driving within the general safe envelope but still safely in the driver's preferred style of driving" (p.2).

Second, research is needed to establish whether novel geometric design principles for turning movements can enable additional capacity gains. For instance, recent work by Kozey and colleagues [51] demonstrates that flexible use of road space enabled by vehicle automation has the potential, under certain circumstances, to allow increased capacity without large increases to the physical footprint of an intersection.

Third, given the context-specificity of whether the "strong" or "weak" Assured Clear Distance Ahead criterion is the standard to which human drivers are held, there is a need for policy clarity as to which of these criteria will govern AVs' driving behavior in various sets of circumstances. When performing turning maneuvers, an argument in favor of the applicability of the more generous "weak" ACDA interpretation is that the leading vehicle in a couplet does not physically block the sight line of the following vehicle to the same degree as when both vehicles are traveling in a straight line [52].

Fourth, further research is required to establish the operations of noncooperative AVs during "start-up" and "clearance" time at the beginning and end of a signal phase, to complement the saturation flow rates calculated in this research for the interior time points of a signal phase. Other important research questions relate to AVs' operations during permitted (as opposed to protected) turning movements and the operations of cooperative (connected) AVs when performing turning maneuvers. With regard to cooperative driving behavior, it will be necessary to establish whether connectivity between vehicles will simply reduce latency times towards zero or will allow exchange of actionable intent information (cf. [53]), the latter of which extends beyond the protocols of the currently planned rollout of vehicle-tovehicle communications [54].

Finally, there is a need for integrated analysis of uncooperative AVs operations across a diversity of roadway geometrics and driving maneuvers. This line of enquiry would follow on from recent research by Shelton et al. [31] that studied connected-vehicle operations on a part-freeway/partsignalized-arterial network. In the coming years, it is likely that AVs operating independently of one another (as partially automated vehicles currently do) will predate the arrival of connected AVs that rely on communications links to external sensors (e.g., V2V/V2I) for making safety-critical decisions. Therefore, planners, road-network managers, and transport policymakers urgently need both standardized techniques and reliable findings regarding the impacts of "unconnected" noncooperative AVs that drive defensively, as human drivers today do.

\section{Summary of Notations}

$\mathrm{veh}_{l}, \mathrm{veh}_{f}$ : Leading and following vehicle, respectively $H, H_{\text {min }}$ : Headway (front of $\mathrm{veh}_{l}$ to front of $\mathrm{veh}_{f}$ ), units of seconds. $H_{\min }$ is minimum value

$H_{\text {min }}^{l, f}: \quad H_{\text {min }}$, conditional on whether $\operatorname{veh}_{l}$ and $\operatorname{veh}_{f}$ perform ABS-engaged or ABS-not-engaged braking maneuvers. For instance, $H_{\min }^{\mathrm{ABS}, \mathrm{noABS}}$ denotes $H_{\min }$ if veh ${ }_{l}$ performs an ABS-engaged braking maneuver and $v^{2} h_{f}$ performs an ABS-not-engaged maneuver

$x, x_{\text {min }}$ : Spacing (front of $\mathrm{veh}_{l}$ to front of $\mathrm{veh}_{f}$ ), units of feet. $x_{\min }$ is minimum value

$v: \quad$ Translational velocity, units of $\mathrm{ft} / \mathrm{sec}$

$\omega: \quad$ Rotational velocity, units of $\mathrm{deg} / \mathrm{sec}$

$a_{l}, a_{f}$ : Rate of deceleration of leading and following vehicle (units of $\mathrm{ft} / \mathrm{sec}^{2}$ ), respectively

$\beta_{l}, \beta_{f}: \quad$ Angle (degrees) at which $\mathrm{veh}_{l}$ and $\mathrm{veh}_{f}$, respectively, initiate emergency braking 
$\alpha: \quad$ Orientation (angle, units of degrees) at rest (at completion of braking maneuver)

$t_{\text {braking: }}$ Duration (seconds) of braking maneuver

$t_{\text {lag, },}$ : Time lag (seconds) between $\mathrm{veh}_{l}$ and $\mathrm{veh}_{f}$ initiating emergency braking. Fixed at 0.4 seconds throughout analysis (see discussion in Section 3.2).

$x_{\text {veh }}$ : Length of vehicles (ft). Fixed at $19^{\prime}$ throughout analysis (see discussion in Section 3.2)

$C_{v}$ : $\quad$ Capacity (vehicle per hour per lane) at velocity $v$

$r$ : $\quad$ Turning radius ( $\mathrm{ft}$ ), defined as radius of vehicle centroid

$v^{\mathrm{WLB}}: \quad$ Maximum speed at which a wheels-locked braking maneuver can be performed without encroaching into oncoming traffic or past the edge-of-lane after exiting the intersection

$v^{\mathrm{HDM}}$ : Average speed of human drivers during turning maneuver, assuming approach-segment speed of $v=35 \mathrm{mph}$ (per [33])

$v^{\mathrm{CTF}}:$ Maximum speed to avoid skidding during turning maneuver (i.e., constrained by tire/roadway-interface friction).

\section{Disclosure}

The usual disclaimer applies: any errors in this paper are the authors' sole responsibility.

\section{Conflicts of Interest}

The authors declare that there are no conflicts of interest regarding the publication of this paper.

\section{Acknowledgments}

The authors wish to thank Catherine Herne for helpful discussions and acknowledge financial support from the National Science Foundation of China (Project no. 71671147) and the University Transportation Research Center, Region 2 (Grant no. 49198-13-28: Simulation of Automated Vehicles' Drive Cycles).

\section{References}

[1] S. Smith, J. Bellone, S. Bransfield et al., "Benefits Estimation Framework for Automated Vehicle Operations," U.S. Dept. of Transportation ITS Joint Program Office Report, FHWA-JPO16-229, 2015.

[2] V. Milanes, S. E. Shladover, J. Spring, C. Nowakowski, H. Kawazoe, and M. Nakamura, "Cooperative adaptive cruise control in real traffic situations," IEEE Transactions on Intelligent Transportation Systems, vol. 15, no. 1, pp. 296-305, 2014.

[3] A. Millard-Ball, "Pedestrians, Autonomous Vehicles, and Cities," Journal of Planning Education and Research, 2016.

[4] R. Anderson, S. Doecke, J. Mackenzie, and G. Ponte, "Potential benefits of autonomous emergency braking based on in-depth crash reconstruction and simulation," in Proceedings of the 23rd International Conference on Enhanced Safety of Vehicles, US National Highway Traffic Safety Administration, 2013.
[5] S. Lam, J. Taghia, and J. Katupitiya, "Evaluation of a transportation system employing autonomous vehicles," Journal of Advanced Transportation, vol. 50, no. 8, pp. 2266-2287, 2016.

[6] NACTO, "NACTO Policy Statement on Automated Vehicles," 2016, http://nacto.org/wp-content/uploads/2016/06/NACTOPolicy-Automated-Vehicles-201606.pdf.

[7] Y. Kong, S. Le Vine, and X. Liu, "Capacity impacts and optimal geometry of automated cars parking facilities," in Proceedings of the Automated Vehicles Symposium, Transportation Research Board and Association for Unmanned Vehicle Systems International, San Francisco, Calif, USA, July 2016.

[8] R. Estepa, A. Estepa, J. Wideberg, M. Jonasson, and A. StenssonTrigell, "More Effective Use of Urban Space by Autonomous Double Parking," Journal of Advanced Transportation, vol. 2017, pp. 1-10, 2017.

[9] A. Malokin, P. L. Mokhtarian, and G. Circella, "How Do Activities Conducted while Commuting Influence Mode Choice? Testing Public Transportation Advantage and Autonomous Vehicle Scenarios," in Proceedings of the Annual Meeting of the Transportation Research Board, Washington, DC, USA, January 2016.

[10] R. Krueger, T. H. Rashidi, and J. M. Rose, "Preferences for shared autonomous vehicles," Transportation Research Part C: Emerging Technologies, vol. 69, pp. 343-355, 2016.

[11] M. Stanley, "Shared mobility on the road of the future," 2016, http://www.morganstanley.com/ideas/car-of-future-is-autonomous-electric-shared-mobility.

[12] M. Gucwa, "Mobility and energy impacts of automated cars," in Proceedings of the Automated Vehicles Symposium, San Francisco, Calif, USA, 2014.

[13] S. Childress, B. Nichols, B. Charlton, and S. Coe, "Using an activity-based model to explore possible impacts of automated vehicles," in Proceedings of the 94th Annual Meeting of the Transportation Research Board, Washington, DC, USA, 2015.

[14] K. Kim, G. Rousseau, J. Freedman, and J. Nicholson, "The Travel Impact of Autonomous Vehicles in Metro Atlanta through Activity-Based Modeling," in Proceedings of the 15th TRB National Transportation Planning Applications Conference, Atlantic City, NJ, USA, 2015.

[15] E. Guerra, "Planning for Cars That Drive Themselves: Metropolitan Planning Organizations, Regional Transportation Plans, and Autonomous Vehicles," Journal of Planning Education and Research, vol. 36, no. 2, pp. 210-224, 2016.

[16] S. Tsao and S. Chu, "A study on adjustment factors for U-turns in left-turn lanes at signalized intersections," Journal of Advanced Transportation, vol. 29, no. 2, pp. 183-192, 1995.

[17] W. T. Hung, F. Tian, and H. Y. Tong, "Discharge headway at signalized intersections in Hong Kong," Journal of Advanced Transportation, vol. 37, no. 1, pp. 105-117, 2003.

[18] Y. Gu, Y. Hashimoto, L.-T. Hsu, M. Iryo-Asano, and S. Kamijo, "Human-like motion planning model for driving in signalized intersections," IATSS Research, vol. 41, no. 3, pp. 129-139, 2017.

[19] Transportation Research Board, Highway Capacity Manual, A Guide for Multimodal Mobility Analysis, Washington, DC, USA, 6th edition, 2016.

[20] R. Hoogendoorn, B. Van Arem, and S. Hoogendoorn, "Automated driving, traffic flow efficiency, and human factors," Transportation Research Record, vol. 2422, pp. 113-120, 2014.

[21] G. Arnaout and S. Bowling, "Towards reducing traffic congestion using cooperative adaptive cruise control on a freeway with a ramp," Journal of Industrial Engineering and Management, vol. 4, no. 4, pp. 699-717, 2011. 
[22] P. A. Ioannou, Automated Highway Systems, Springer, New York, NY, USA, 1997.

[23] U. Karaaslan, P. Varaiya, and J. Walrand, "Two proposals to improve freeway traffic flow," in Proceedings of the American Control Conference, pp. 2539-2544, IEEE, June 1991.

[24] J. Vander Werf, S. E. Shladover, M. A. Miller, and N. Kourjanskaia, "Effects of adaptive cruise control systems on highway traffic flow capacity," Transportation Research Record, vol. 1800, no. 1, pp. 78-84, 2002.

[25] B. Van Arem, C. J. G. Van Driel, and R. Visser, "The impact of cooperative adaptive cruise control on traffic-flow characteristics," IEEE Transactions on Intelligent Transportation Systems, vol. 7, no. 4, pp. 429-436, 2006.

[26] K. Dresner and P. Stone, "A multiagent approach to autonomous intersection management," Journal of Artificial Intelligence Research, vol. 31, pp. 591-656, 2008.

[27] M. Ferreira and P. M. D'Orey, "On the impact of virtual traffic lights on carbon emissions mitigation," IEEE Transactions on Intelligent Transportation Systems, vol. 13, no. 1, pp. 284-295, 2012.

[28] Z. Li, L. Elefteriadou, and S. Ranka, "Signal control optimization for automated vehicles at isolated signalized intersections," Transportation Research Part C: Emerging Technologies, vol. 49, pp. 1-18, 2014.

[29] S. Le Vine, A. Zolfaghari, and J. Polak, "Autonomous cars: The tension between occupant experience and intersection capacity," Transportation Research Part C: Emerging Technologies, vol. 52, pp. 1-14, 2015.

[30] S. Le Vine, X. Liu, F. Zheng, and J. Polak, "Automated cars: Queue discharge at signalized intersections with "AssuredClear-Distance-Ahead" driving strategies," Transportation Research Part C: Emerging Technologies, vol. 62, pp. 35-54, 2016.

[31] J. Shelton, S. Samant, J. Wagner, G. Goodin, E. Seymour, and T. Lomax, Revolutionizing Our Roadways: Modeling the Traffic Impacts from Automated and Connected Vehicles in a Complex, Congested Urban Setting, Texas A\&M Transportation Institute, 2016.

[32] C. Katrakazas, M. Quddus, W. H. Chen, and L. Deka, "Real-time motion planning methods for autonomous on-road driving: State-of-the-art and future research directions," Transportation Research, vol. 60, pp. 416-442, 2015.

[33] W. K. M. Alhajyaseen, M. Asano, H. Nakamura, and D. M. Tan, "Stochastic approach for modeling the effects of intersection geometry on turning vehicle paths," Transportation Research Part C: Emerging Technologies, vol. 32, pp. 179-192, 2013.

[34] A. Wolfermann, W. K. Alhajyaseen, and H. Nakamura, "Modeling speed profiles of turning vehicles at signalized intersections," in Proceedings of the 3rd International Conference on Road Safety and Simulation, Indianapolis, Ind, USA, 2011.

[35] S. Cooner, S. Ranft, Y. Rathod et al., "Development of guidelines for triple left and dual right-turn lanes," Tech. Rep. 0-6112-1, Texas Transportation Institute, 2011.

[36] T. Sando and R. N. Mussa, "Site Characteristics Affecting Operation of Triple Left-Turn Lanes," Transportation Research Record, vol. 1852, pp. 55-62, 2003.

[37] K. Courage, B. Stephens, A. Gan, and M. Willis, Triple LeftTurn Lanes at Signalized Intersections. Florida Department of Transportation, 2017, http://www.fdot.gov/research/completed proj/summary_te/fdot_bc131rpt.pdf.

[38] J. D. Leonard, "Operational characteristics of triple left turns," Transportation Research Record, vol. 1457, pp. 104-110, 1994.
[39] K. W. Ackeret, "Criteria for the geometric design of triple leftturn lanes," ITE Journal, vol. 64, no. 12, pp. 27-33, 1994.

[40] J. D. Buchwalter, W. H. Danne, J. J. Dvorske et al., American Jurisprudence: Automobiles and Highway Traffic, 2nd edition, 2016.

[41] J. E. Naffky, Corpus Juris Secundum: A Contemporary Statement of American Law as Derived from Reported Cases and Legislation, vol. 60A, Motor Vehicles, 2012.

[42] "Louisiana Farm Bureau Mutual Insurance v. Dunn. 484 So.2d 853," 1986

[43] S. Shladover, D. Su, and X. Lu, "Impacts of Cooperative Adaptive Cruise Control on Freeway Traffic Flow," Transportation Research Record, vol. 2324, pp. 63-70, 2012.

[44] J. F. Stanek and J. A. Lockwood, "Automotive Drone Deployment System. United States Patent Application \#2016/0347452," Assigned to Ford Global Technologies LLC, 2016.

[45] American Association of State Highway and Transportation Officials, A Policy on Geometric Design of Highways and Streets, 6th edition, 2011.

[46] D. B. Fambro, K. Fitzpatrick, and R. J. Koppa, "Determination of Stopping Sight Distances," National Cooperative Highway Research Program (NCHRP) Report, 400, National Academy Press, Washington DC, USA, 1997.

[47] W. Hulshof, I. Knight, A. Edwards, M. Avery, and C. Grover, "Autonomous Emergency Braking Results", in Proceedings of the 23rd International Conference on Enhanced Safety of Vehicles, US National Highway Traffic Safety Administration, Washington, DC, USA, 2013.

[48] D. Burton, A. Delaney, S. Newstead, D. Logan, and B. Fildes, Effectiveness of ABS and Vehicle Stability Control Systems. ISBN, Royal Automobile Club of Victoria, Victoria, Australia, 2004.

[49] National Highway Traffic Safety Administration, Final Report for the Methodology study of the Consumer Braking Information Initiative - Phase II, 2000.

[50] C. P. Urmson, D. A. Dolgov, and P. Nemec, "Driving Pattern Recognition and Safety Control," United States Patent 8634980, 2014.

[51] P. Kozey, Y. Xuan, and M. J. Cassidy, "A low-cost alternative for higher capacities at four-way signalized intersections," Transportation Research Part C: Emerging Technologies, vol. 72, pp. 157-167, 2016.

[52] C. de Santos-Berbel, M. Essa, T. Sayed, and M. Castro, "Reliability-Based Analysis of Sight Distance Modelling for Traffic Safety," Journal of Advanced Transportation, vol. 2017, Article ID 5612849, 12 pages, 2017.

[53] A. C. Mersky and C. Samaras, "Fuel economy testing of autonomous vehicles," Transportation Research Part C: Emerging Technologies, vol. 65, pp. 31-48, 2016.

[54] J. Harding, G. Powell, R. Yoon et al., Vehicle-to-Vehicle Communications: Readiness of V2V Technology for Application, Report No. DOT HS 812 014, National Highway Traffic Safety Administration, Washington, DC, USA, 2014. 


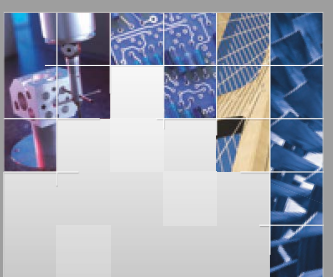

\section{Enfincering}
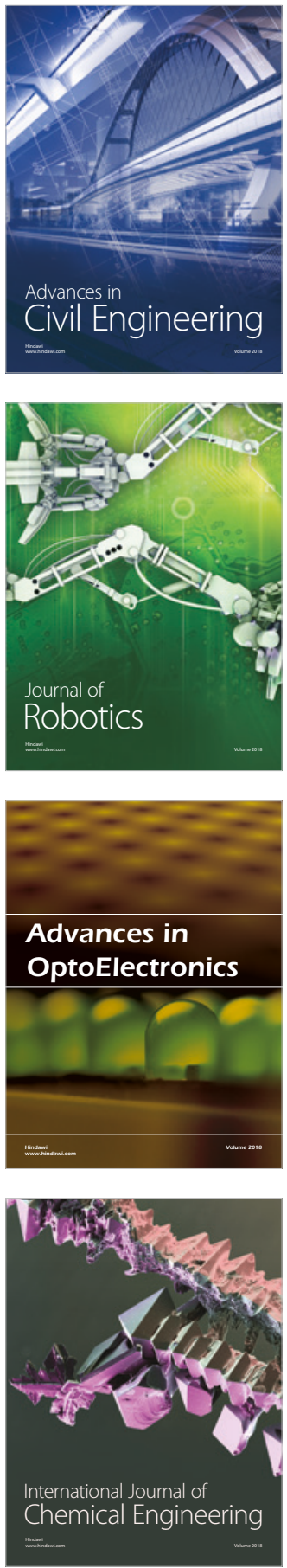

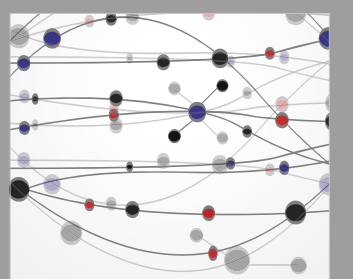

\section{Rotating \\ Machinery}

The Scientific World Journal

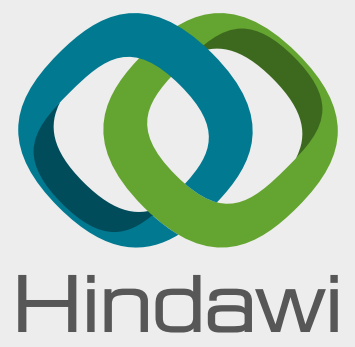

Submit your manuscripts at

www.hindawi.com
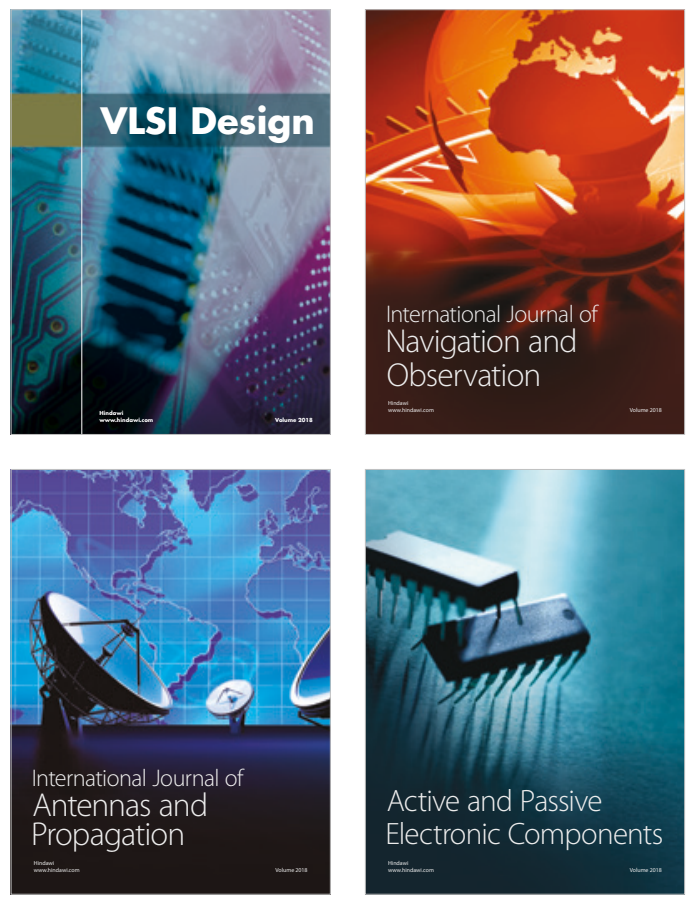
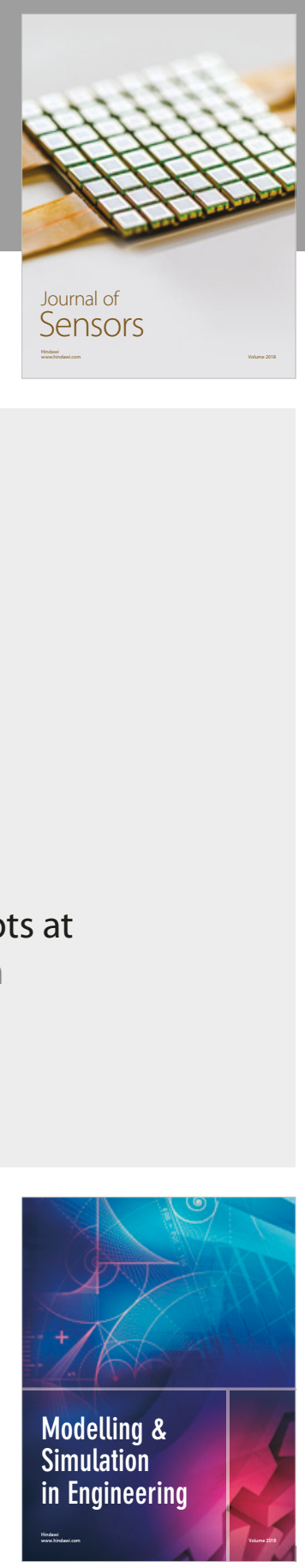

\section{Advances \\ Multimedia}
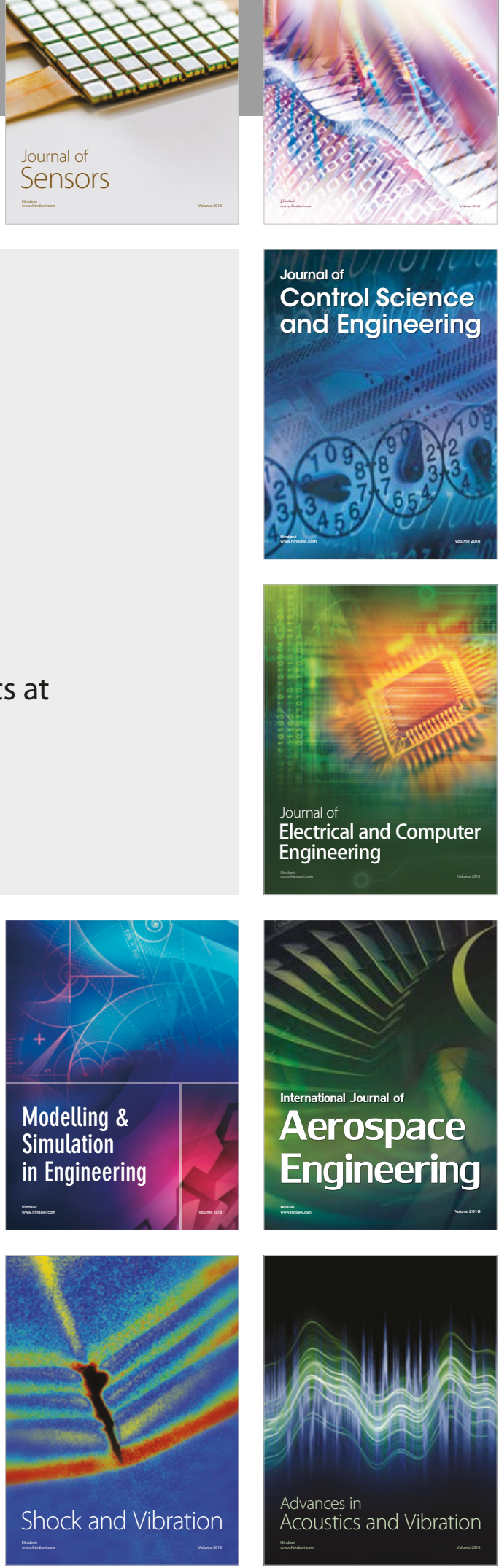